\title{
Classification of EEG signal using EACA based approach at SSVEP-BCI
}

\author{
Ashwini S. R. ${ }^{1}$, H. C. Nagaraj ${ }^{2}$ \\ ${ }^{1}$ Department of Telecommunication Engineering, JNN College of Engineering, India \\ ${ }^{2}$ Department of Electronics \& Communication Engineering, Nitte Meenakshi Institute of Technology, India
}

\begin{abstract}
Article Info
Article history:

Received Jan 7, 2021

Revised Apr 20, 2021

Accepted May 4, 2021

\section{Keywords:}

Brain computer interfaces Electroencephalography

Event based adaptive component approach Information transfer rate Steady state visually evoked potentials

ABSTRACT

The brain-computer-interfaces (BCI) can also be referred towards a mindmachine interface that can provide a non-muscular communication channel in between the computer device and human brain. To measure the brain activity, electroencephalography (EEG) has been widely utilized in the applications of BCI to work system in real-time. It has been analyzed that the identification probability performed with other methodologies do not provide optimal classification accuracy. Therefore, it is required to focus on the process of feature extraction to achieve maximum classification accuracy. In this paper, a novel process of data-driven spatial has been proposed to improve the detection of steady state visually evoked potentials (SSVEPs) at BCI. Here, EACA has been proposed, which can develop the reproducibility of SSVEP across many trails. Further this can be utilized to improve the SSVEP from a noisy data signal by eliminating the activities of EEG background. In the simulation process, the SSVEP dataset recorded from given 11 subjects are considered. To validate the performance, the state-of-art method is considered to compare with the EDCA based proposed approach.
\end{abstract}

This is an open access article under the CC BY-SA license.

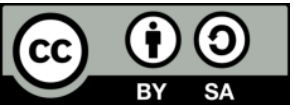

\section{Corresponding Author:}

Ashwini S. R.

Department of Telecommunication Engineering

JNN College of Engineering

Navule, Sawalanga Road, Shivamogga, 577204, Karnataka, India

Email: srashwini@jnnce.ac.in

\section{INTRODUCTION}

The technical advancement in biomedical and neuroscience has enabled the direct communication channel among computers and the human brain. In the brain, the electrical activity is produced by the help of neuronal postsynaptic membrane polarity changes which can be monitored to recognize patient intentions [1]. Brain-computer-interfaces (BCIs) can give a novel communication channel to the humans and have received huge attention. The BCIs use signals, which is generated by the central-nervous system and primary target is severe neuromuscular disorders (such as spinal cord injury, amyotrophic lateral sclerosis, cerebral palsy, and brain-stem stroke). The advanced BCI methods provide healthy people with alternative way of communication, control, and security [2]-[4]. Hence, these methods have evolved promising in the applications of body area network [5]-[8]. In order to measure the activity of the brain, the electroencephalography (EEG) is widely employed in the applications of BCI due to its higher activity as it is necessary for BCIs to work as the system in real-time [9]. Additionally, the devices of EEG are portable and inexpensive.

In general, the steady state visual evoked potential (SSVEP) signal is like sinusoidal waveforms and appear at a similar fundamental frequency as that of stimulus and its harmonics [10]. Anyways, the 
oscillations are spontaneous and are unrelated to stimulation and exist in EEG recordings. Thus, an algorithm of robust detection is needed to construct the system of reliable BCI. In last few decades, several algorithms have been introduced for SSVEP [11]-[17]. The power spectral density analysis (PSDA) approach can be considered since the features of distinctive SSVEPs can be observed in the domain of frequency [11]. Anyways, PSDA is known to be susceptible to noise and the long durations are required to maximize the signal to noise ratio (SNR). A canonical correlation analysis (CCA), multivariable statistical method (MSM) [12], [14] exploits the multiple channels of covariance information to improve the SNR and give much better recognition accuracy compared to the PSDA. In the SSVEP identification research, high robustness, simple implementation, and good performance have made the CCA attractive. On the other side, the CCA is insufficient to remove the information of discrimination that is embedded in harmonic features of filter-bank canonical correlation analysis (FBCCA) and SSVEPs are introduced to manage this problem [15]. FBCCA uses distinct spectral properties of more than one harmonic frequency and neglects correlation information among SSVEP responses at various frequencies [16]. Moreover, these methods neither consider all stimuli bandwidth nor fully maximize SSVEP potential based on BCIs. Hence, it is essential to utilize the SSVEP model in the identification algorithm. Here, identification probability performed by the help of other methodology is analyzed and found that it does not provide optimum classification accuracy. Therefore, it is required to focus on the process of feature extraction (FE) to achieve maximum accuracy of classification. In this paper, a novel data-driven spatial process is developed to improve the detection of SSVEPs at BCI. EACA is proposed that can develop the reproducibility of SSVEP across many trails and can be utilized to improve the SSVEP as an SNR data signal by removing the activities of EEG background. The event based adaptive component analysis (ACA) is an approach that can remove event features effectively by means of maximizing the regeneration at a certain period of the task. Here, two signals are considered: event unrelated and event-related signals. Furthermore, EACA filters is introduced to integrate with more than one frequency simulation that can develop the EACA version to optimize the process of FE. In the stage of classification, the PSD is utilized to achieve a higher magnitude for the stimulation frequency. The performance of the proposed model is evaluated by the SSVEP dataset recorded from given 11 subjects [18]. The 11 volunteers have contributed while all are present as the staff of "Centre for Research and Technology, Hallas" (CERTH). Specifically, 8 of them were males and 3 were females with their ages around 25-39 years. In order to estimate the performance, the state-of-art outcomes is utilized and compared with this EDCA based proposed approach.

In the recent years, several researchers have focused on developing an EEG based BCI for medical applications in order to maximize the life quality. Moreover, the BCI can be used in the field of entertainment, gaming, and marketing to provide the users with some extra-personalized experiences; both the non-medical and medical application need the capability to interpret user's emotional experience and multimedia-induced perception. The BCI process allows to collaborate with various application through direct connection at brain and output devices by brain signals. A variety of technologies can be used to acquire the brain signal such as electrocorticography ('ECoG'), magnetoencephalography ('MEG'), functional near-infrared spectroscopy ('fNIRS'), electroencephalography, functional magnetic-resonance imaging ('fMRI') and so on. Amongst these, EEG is a most extensively used technique due to the high-temporal resolution and economic efficiency. Yet, the predictable EEG based BCIs are very uncomfortable for practical application as it contains several numbers of EEG electrodes and wearing EEG-cap calls for skilled assistants. Therefore, ear-EEG-BCIs has been in research for convenient BCI, where the ear-EEG can be separated into the measuring EEG signals that are observed around the 'outer ear' or the 'in ear'. The study of ear-EEG-BCI research generally targets the auditory/visual stimuli system or at the drowsiness detection scheme. It has been observed that there are very less studies that used motor-imagery detection approach based on the ear-EEG. Motor-imagery detection is the normally used model in the BCI due to its characteristics which does not depends on any external stimuli and also motor-imagery associated with the ear-EEG can provide a useful BCI applications to the real-world. Therefore, Kim et al. [19] has discussed about the investigation feasibility of motor-imagery classification based on ear-around-EEG signals. BCI can also be referred towards a brain-machine interface ('BMI'), or mind-machine interface ('MMI') that can provide a non-muscular communication channel in between the computer device and human brain. The progression in computer interface and low-cost electronics with the requirement to serve suffering people from such neuromuscular disabilities is a novel field of research that has emerged like the different brain function. The EEG is an electrically active signal generated by the brain and that is recorded from surface of the scalp through electrodes. The researchers mainly depend on EEG in order to characterize the activity of brain, as this is recorded noninvasively through portable equipment. EEG or the activity of brain generally is used in real time to regulate external devices by a broad BCI system. However, in [20], a typical BCI structure is proposed that generally consists of an approach of data acquisition, pre-processing step at the acquired signals, feature extraction method, features classification, post-processing method for output

Int J Artif Intell, Vol. 10, No. 3, September 2021: 717 - 726 
classifier, and lastly the device controller and control interface. In the post-processing step, the output signals are usually translated into suitable commands to control the output devices, by several applications like as video games, robotic arms, and wheelchair.

The appropriate classification of EEG signals is essential for the improvement of consistent motor imagery (MI) BCI system. Qureshi et al. [21], publicly accessible BCI Competition-IV (dataset IIa) is utilized. Additionally, the difficulty in binary classification of motor imagery EEG signal data through using sigmoid initiation function is addressed that depends upon the extreme learning machine (ELM). Thus, it specifies the need for a novel approach of extracting features from captured EEG signal by applying the independent component analysis (ICA) over a period of series data. This further transforms an ICA epoch series data into the Fourier domain process and then used to extract the information of phase from using Fourier spectrum. Therefore, the information of phase is further used to compute the increased crosscorrelation matrix connectivity. Also, the upper diagonal matrix is further useful in order to serve the basic feature at the classification framework of ELM. Emotions are the significant features that helps in the interaction among different people and thereby play an important role in the human life. It motivates the human to respond to the stimuli in the environment which helps in the improvement of the communication and the decision-making. The automatic-emotion recognition is an interesting area from the past decade, due to the increasing BCI in the interaction between user and computer. The emotion-recognition can be carried out from the facial-expressions, gestures, the speech as well as text. These can be recorded in many ways like electroencephalography ('EEG'), magnetic resonance imaging ('MRI'), and positron emission tomography ('PET'). Few emotional states namely the fear, sad, happy, frustrated and pleasant' feature extraction and classification are done from the inner-emotion EEG signals in [22]. Y1ldirım and Varol [23] discussed the emotional-recognition by using the EEG signals and the brain-computer-interface studies. EEG provides the monitoring of the electrical activities of the brain. BCI can convert the electrical signals that is produced by the brain to the signals that can be interpreted by the computer or any other electronic systems. The aim of this is to produce the results developed by the computer-system and compared to response in the body from the signals that are sent from the brain. The emotions are psychophysiological changes in the moods of the person that will emerge by the interactions with the bio-chemical and the environmental effects. There are various on-going researches on the emotion recognition [24]. Some of the researches include the voicerecognition, and the recognition with the mimics. The facial expression or the tone of the voice might not always represent the feelings. Extra factors are also used which include the skin-conductivity, the changes in the skin due to temperature, the blood-pressure, and the heart-beat for the estimation of emotions. Thus BCIresearch is significant in the recent years and still a new topic in the research field.

\section{RESEARCH METHOD}

\subsection{Target detection methodology}

Here, a novel data-driven spatial method of approach is used for optimized SSVEPs detection. The EACA based method is represented with distinct data standardization to identify target identification and to improve the SS-VEP SNR data signal via removing the activities of EEG background. Furthermore, the proposed model of EACA filters integrate with more than one frequency simulation that can improve the EACA version to optimize the process of feature extraction. Figure 1 shows the system diagram of an SSVEP-based BCI. Each standardization data at $\mathrm{a}^{\text {th }}$ detection and single trial test data is represented in terms of 4-dimensional tensor as (1).

$$
B=(B)_{a c z y} \in H^{E_{d} \times E_{f} \times E_{g} \times E_{k}}
$$

Whereas, the 2-dimensional tensor is given as (2):

$$
B \in H^{E_{f} \times E_{g}}
$$

The detection index is represented by $Q, E_{d}$ will represent the number of detections, $c$ shows channel index, $E_{f}$ shows channel number, $z$ indicates the index of sample point and $E_{g}$ is number of sampling point in each trail. $E_{k}$ is the number of training trails and $y$ indicates the index of training trail. The target detection has $B$ inputs and allocates to $E_{d}$ classes with $K_{e}$ stimulation frequency. Then $J_{e}$ can be written as (3):

$$
J_{e} \in\left\{J_{1}, J_{2}, J_{3}, \ldots, J_{E_{d}}\right\}
$$

The filter bank analysis is applied to decompose SS-VEP into components of sub-band, therefore the information of independent component present at the component of harmonics will be removed more 
effectively [24]. In accordance with the paper [24], the upper and lower cut-off frequencies of $l-t h$ sumband is set to $90 \mathrm{~Hz}$ and $l \times 8 \mathrm{~Hz}$, respectively. Thereafter, $l-t h$ pass-band ripple filter with zero phase is applied that depends on infinite impulse response (IIR) system. For this, the training and testing data can be given as (4):

$$
\begin{aligned}
& B^{(l)} \in H^{E_{d} \times E_{f} \times E_{g} \times E_{k}} \\
& B^{(l)} \in H^{E_{f} \times E_{g}}
\end{aligned}
$$

In order to match the template based on target identification framework, the feature values dependent correlation values for $a-t h$ detection and $l-t h$ sub-band can be computed through,

$$
m_{a}^{l}=J\left(B^{(l)}, B^{(l)}\right)
$$

Moreover, the weighted sum of squares of the collective values of correlation conforming to all consider subband components that can be computed as the features at target detection is given by (6):

$$
N_{a}=\sum_{l=1}^{P_{l}} Q(l) \times\left(m_{a}^{l}\right)^{2}
$$

where total number of 'sub-bands' is represent by $P_{l}$ and value of $Q(l)$ can be computed as (7):

$$
Q(l)=\frac{1}{l^{5 / 4}}+\frac{1}{4}
$$

The targeted class $\left(K_{r}\right)$ can be acknowledged through by (8):

$$
r=\arg _{a} \max N_{a}, n=1,2,3, \ldots, E_{d}
$$

In the model of SSVEP-BCIs, it is required to obtain the optimized FE method $J($.$) to improve the accuracy$ of target detection.

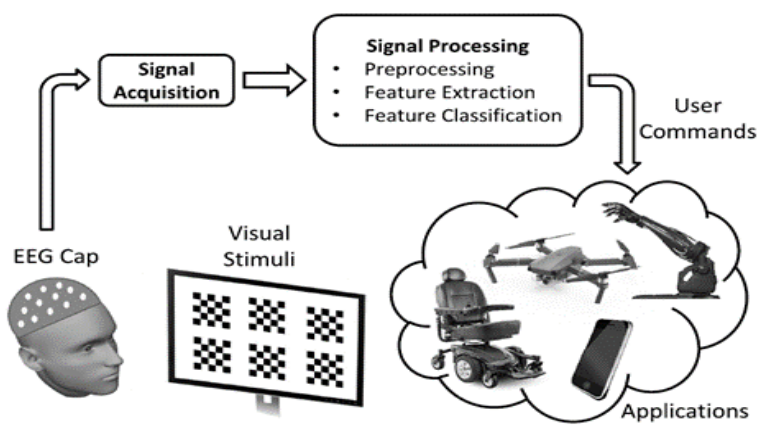

Figure 1. System diagram of an SSVEP-based BCI

\subsection{Proposed EACA based approach}

The event-based ACA is an approach that can remove the components of the event occurred via maximizing regeneration at a certain time. There are two types of signals; event related and unrelated signals $(U(t) \in H$ and $V(t) \in H)$. A linear generative prototype of computed multi-channel EEG signal $R(t) \in H^{E_{f}}$ is presented as (9).

$$
R_{c}(t)=Q_{1, c} U(t)+Q_{2, c} V(t), \text { and } c=1,2,3, \ldots, E_{f}
$$

where c denotes the channel index, $Q_{1, c}$ and $Q_{2, c}$ shows the mixing component and project the signals source to EEG signals. However, the difficulty arises at discarding the event associated component from detected signal summation $R(t)$. 


$$
\begin{aligned}
& M(t)=\sum_{c=1}^{E_{f}} R_{c}(t) X_{c} \\
& M(t)=\sum_{c=1}^{E_{f}}\left(Q_{1, c} U(t) X_{c}+Q_{2, c} V(t) X_{c}\right)
\end{aligned}
$$

Preferably, the solution can be given in such a way where, $\sum_{c=1}^{E_{f}} Q_{1, c} X_{c}=1$ and $\sum_{c=1}^{E_{f}} Q_{2, c} X_{c}=$ 0 and leads to final outcome $U(t)=M(t)$. The difficulty can be minimized by maximization of inter-event covariation; $\mathrm{y}^{\text {th }}$ event of EEG signal and related component of predicted event are denoted as $R^{(y)}(t)$ and $M^{(y)}(t),\left(y=1,2,3, \ldots, E_{k}\right)$. Here, $M^{(y)}(t)$ epoch is stable where $t \in\left[t_{y}, t_{y}+T\right]$, and ' $T^{\prime}$ denotes the interval of each task event. The covariation of events $y_{1}$ and $y_{2}$ at $M(t)$ are given as (12), (13):

$$
\begin{aligned}
& S_{y_{1}, y_{2}}=\operatorname{Cov}\left(M^{\left(y_{1}\right)}(t), M^{\left(y_{2}\right)}(t)\right) \\
& S_{y_{1}, y_{2}}=\sum_{c_{1}, c_{2}=1}^{E_{f}} X_{c_{1}} X_{c_{2}} \operatorname{Cov}\left(R_{c_{1}}^{\left(y_{1}\right)}(t), R_{c_{2}}^{\left(y_{2}\right)}(t)\right)
\end{aligned}
$$

Also, the combination of all probable events is summed as (14).

$$
\sum_{\substack{y_{1}, y_{2}=1 \\ y_{1} \neq y_{2}}}^{E_{k}} S_{y_{1}, y_{2}}=\sum_{\substack{y_{1}, y_{2}=1 \\ y_{1} \neq y_{2}}}^{E_{k}} \sum_{c_{1}, c_{2}=1}^{E_{f}} X_{c_{1}} X_{c_{2}} \operatorname{Cov}\left(R_{c_{1}}^{\left(y_{1}\right)}(t), R_{c_{2}}^{\left(y_{2}\right)}(t)\right)=X^{T} W X
$$

Here, the $W$ matrix is given by (15):

$$
W_{c_{1}, c_{2}}=\sum_{\substack{y_{1}, y_{2}=1 \\ y_{1} \neq y_{2}}}^{E_{k}} \operatorname{Cov}\left(R_{c_{1}}^{\left(y_{1}\right)}(t), R_{c_{2}}^{\left(y_{2}\right)}(t)\right)
$$

The $M(t)$ variance is constrained to get finite solution,

$$
\operatorname{Var}(M(t))=\sum_{c_{1}, c_{2}=1}^{E_{f}} X_{c_{1}} X_{c_{2}} \operatorname{Cov}\left(R_{c_{1}}(t), R_{c_{2}}(t)\right)=X^{T} A X
$$

where $X^{T} A X=1$ resolves the constrained optimization difficulty as (17):

$$
\hat{X}=\arg _{X} \max \frac{X^{T} W X}{X^{T} A X}
$$

The coefficient vector is acquired as $\frac{W}{A}$ Eigen-vector matrix. Also, the Eigen values and Eigen vectors are acquired from the matrix $E_{f} \times E_{f}$, where $D$ represents the Eigen value of $\frac{W}{A}$ matrix. It is acquired in a down order where the value of cost function is similar to $\hat{X}$ Eigen vector and the Eigen-values signifies the task consistency among the events. Here, the proposed methodology is also capable to design the spatial filters in order to remove EEG background activity at recording process from scalp. The spatial filters for $\mathrm{a}^{\text {th }}$, simulates the $X_{a}^{(l)} \in H^{E_{f}}$ which is obtained by EACA from an individual standardization data $B_{a}^{(l)}$ by applying the process of filter bank. In (16), Q is computed with concentrated matrix of training trails $B_{a}^{(l)}$ and the correlation coefficient between $B^{(l)} \in H^{E_{f} \times E_{g}}$ single trail test data and average data in training follow through $\mathrm{a}^{\text {th }}$ visual stimulus and is computed as (18): 


$$
m_{a}^{l}=N\left(\left(B^{(l)}\right)^{T} X_{a}^{(l)},\left(\bar{B}_{a}^{(l)}\right)^{T} X_{a}^{(l)}\right)
$$

where $N$ shows the analysis of Pearson correlation between signals. In addition, there are individual calibrating data $E_{d}$ which corresponds to the visual stimuli and different spatial filter $E_{d}$ obtained. Therefore, the integration of spatial filter $X^{(l)} \in H^{E_{d} \times E_{f}}$ is computed as:

$$
X^{(l)}=\left[\begin{array}{llll}
X_{1}^{(l)} & X_{2}^{(l)} & \cdots & X_{E_{d}}^{(l)}
\end{array}\right]
$$

So, (18) is modified in (20).

$$
m_{a}^{l}=N\left(\left(B^{(l)}\right)^{T} X^{(l)},\left(\bar{B}_{a}^{(l)}\right)^{T} X^{(l)}\right)
$$

where $N$ shows the analysis of two-dimensional correlation between signals. After acquisition of $N_{a}$ final features, it is merged to correlation coefficients. Also, it corresponds to existing sub-band components and projected target is detected using (8).

\section{RESULTS AND DISCUSSION}

In this section, publicly accessible dataset is utilized and the ITR and classification accuracy of EEG signal data through EACA based proposed approach is provided. This approach is used to extract features and thereafter perform classification of EEG signal. The code simulation has been done in Matlab 2018a under a system configuration of Intel-i5 processor and 12 GB RAM with windows 10 operating system. The performance of the proposed model is evaluated by the SS-VEP dataset recorded from given 11 subjects [18], where 11 volunteers have contributed who are the staff of "Centre for Research and Technology, Hallas" (CERTH). Specifically, 8 of them were male and 3 of them were female and their ages around 25-39 years. Detailed information is as given in Table 1.

Table 1. Subjects' general information

\begin{tabular}{cccccc}
\hline Subject ID: & Gender & Age & Net Size & Handedness & Hair Type \\
\hline 1 & Male & 24 & Adult Medium & Right & Regular \\
2 & Male & 37 & Adult Small & Right & Regular \\
3 & Male & 39 & Adult Medium & Right & Thick \\
4 & Male & 31 & Adult Medium & Left & Short \\
5 & Female & 27 & Adult Medium & Right & Thick \\
6 & Female & 28 & Adult Medium & Right & Regular \\
7 & Male & 26 & Rdult Medium & Right & Thick \\
8 & Female & 31 & Adult Medium & Right & Short \\
9 & Male & 29 & Adult Medium & Right & Regular \\
10 & Male & 37 & Adult Medium & Adult Medium & \\
11 & Male & 25 & & &
\end{tabular}

A 22-inches LCD monitor has been considered to display visual detection with pixel resolution of 1680x1080. The visual simulation experiment was programmed by using the MS Visual Studio 2010 and OpenGL, where Nvidia GeForce graphic card (GC) were used to offer the faster speed to accommodate more frames in a screen. In addition, vertical synchronization of Nvidia GC was considered to verify receiving frames on the screen. The experimental stimulus which was shown on monitor were changing with different frequencies such as: $6.66 \mathrm{~Hz}, 7.50 \mathrm{~Hz}, 8.57 \mathrm{~Hz}, 10.00 \mathrm{~Hz}$ and $12.00 \mathrm{~Hz}$. The box was wavering with specified frequency and was available for five seconds. This is denoted as trial/event with 5-seconds break at 'visual stimulation' between displays and the box flashes again with different frequency. Background screen was considered to be black throughout the performed experiment with each session having 23-trials. To validate the performance, the state-of-art is considered to compare with the proposed EDCA based proposed approach.

The optimal existing system (OES) result from [25] is considered. Spearman correlation has been used to find the monotonic association between features by considering them as sequences. Therefore, it discounts the absolute changes between the values and depends upon statistical dependent features. Moreover, it can be particularly useful for different subjects and each one has different response for presence 
or absence of the external stimuli. Lastly, based upon the obtained outcome, the SVM classifier is used with a kernel of Spearman correlation, which was chosen to be optimal configuration in [25]. Here, the ITR and accuracy are computed by cross-validation process where $90 \%$ of the data is used for training and rest $10 \%$ data for testing. From Figures 2 to 5, sub-band (SB) one and sampling rate of 250 with varying data-length from $200 \mathrm{~ms}$ to $600 \mathrm{~ms}$ has been fixed. Figures 2 and 3 show the average classification accuracy, where Figure 2 shows averaged classification accuracy as per trails as a function of data length and Figure 3 shows averaged classification accuracy as per subjects as a function of data length.

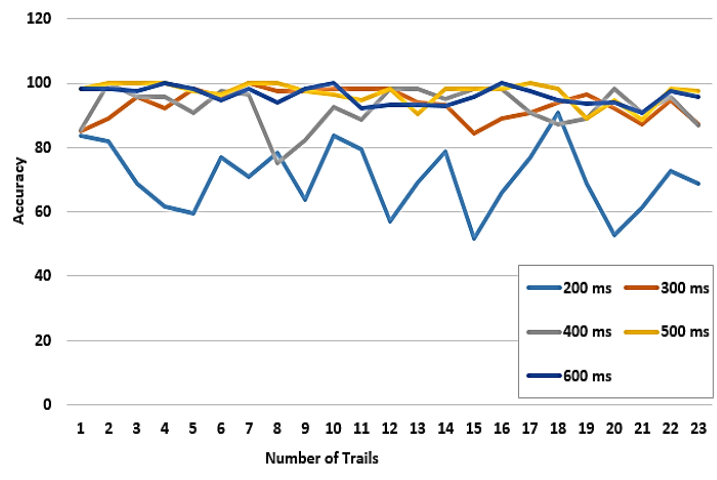

Figure 2. Averaged classification accuracy as per trail across subjects (i.e., w.r.t different data length)

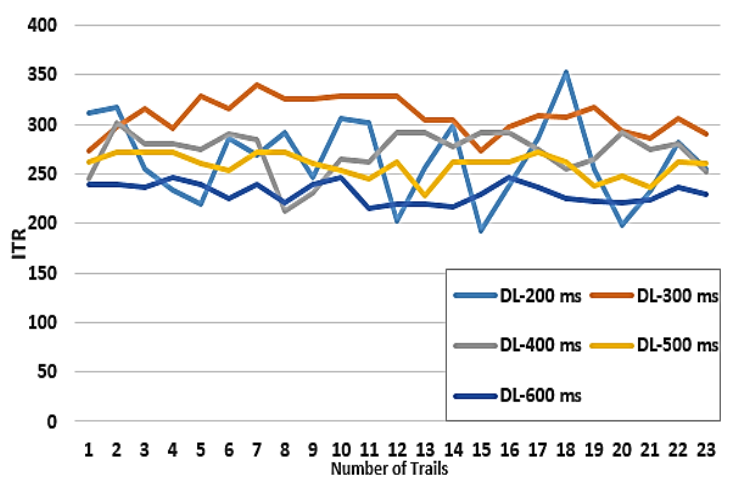

Figure 4. Simulated ITRs as per trails (i.e., w.r.t data length)

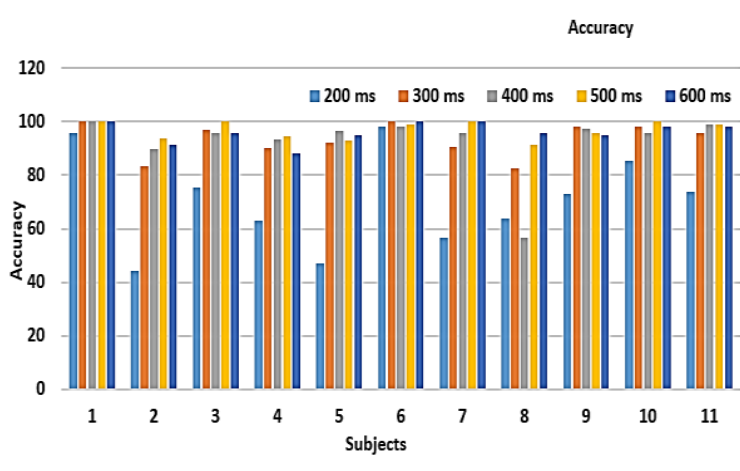

Figure 3. Averaged classification accuracy (i.e., w.r.t different data length)

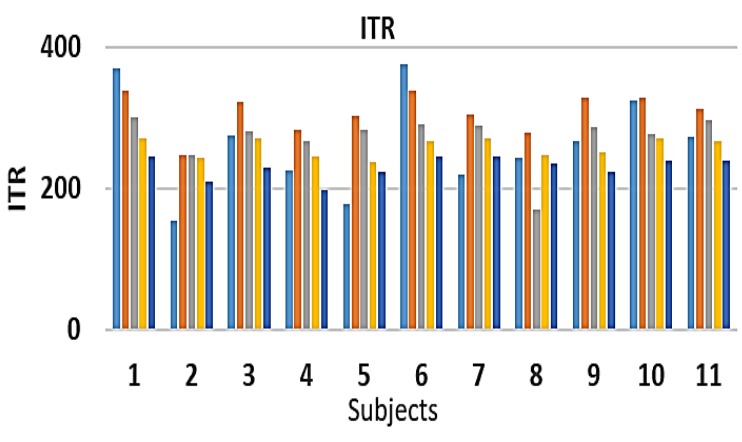

口DL-200 ms $\square \mathrm{DL}-300 \mathrm{~ms} \square \mathrm{DL}-400 \mathrm{~ms} \square \mathrm{DL}-500 \mathrm{~ms} \square \mathrm{DL}-600 \mathrm{~ms}$

Figure 5. Simulated ITRs across subjects different (i.e., w.r.t different data length)

The variations in accuracy can be clearly noted that more than 200ms data length (DL) has always good classification accuracy as per trails and subjects. In addition, ITRs are calculated as per trails and subjects shown in Figures 4 and 5, where DL varies from 200ms to $600 \mathrm{~ms}$ is considered with interval of $100 \mathrm{~ms}$. It is analyzed that in maximum number of trails and subjects, the ITRs range at $300 \mathrm{~ms}$ is much better compared to other considered DL. Figures 6 to 9 have DL of 300ms and sampling rate of 250 with varying SB from 1 to 5 at interval of 1. Figure 6 shows the averaged classification accuracy as per trails as a function of SB and Figure 7 shows the averaged classification accuracy as per subjects as a function of SB. It has been analyzed that, when more SB are considered, classification accuracy is higher. Similarly, Figure 8 shows the ITRs computation as per trails as a function of SB and Figure 7 shows ITRs computation as per subjects as a function of SB. ITRs at more SB provide better results as compared to lower SB. Figures 10 and 11, DL of $300 \mathrm{~ms}$, sampling rate of 250 and SB of 1 , shows the averaged classification accuracy and simulated ITRs across subjects, where at subject 8 and 2 it is noted that, this classification accuracy is lesser to $90 \%$ otherwise very good classification accuracy has been achieved. Whereas, ITR at subjects 2, 4 and 8 have below 300 ITRs otherwise at every subject the proposed model has performed considerably well. Table 2 shows results comparison with respect to state-of-art techniques from [25], where classification accuracy of decision trees, ensemble approach, linear discriminant analysis (LDA), OES and support vector machine $(\mathrm{SVM})$ is shown as per the subject ID. 


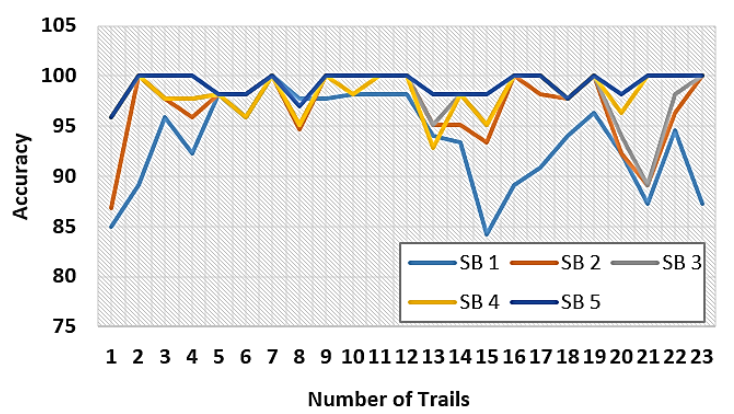

Figure 6. Averaged classification accuracy as per trails (i.e., w.r.t different sub-band)

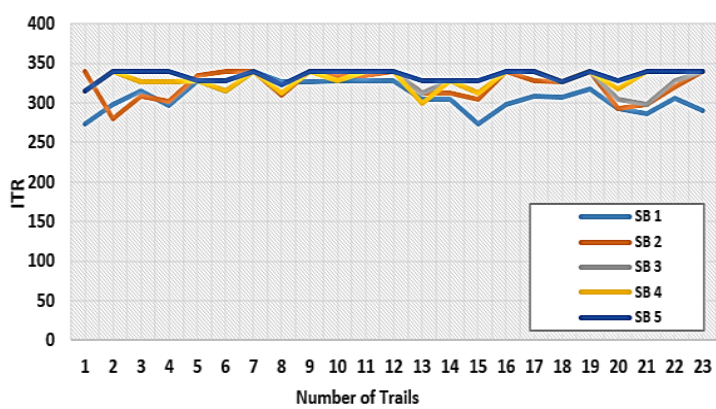

Figure 8. Simulated ITRs as per trails (i.e., w.r.t different sub-band)

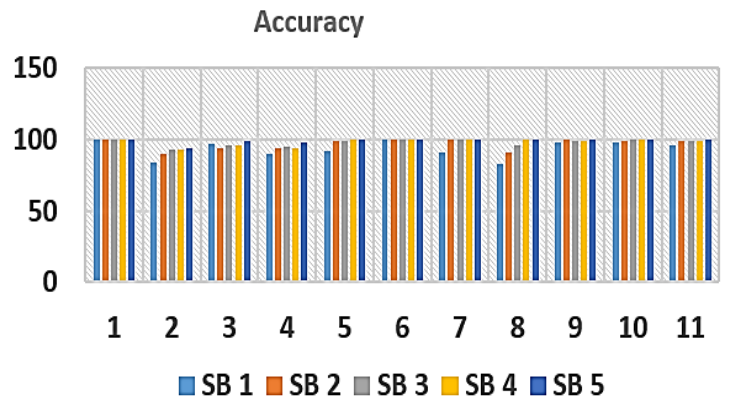

Figure 7. Averaged classification accuracy across subjects (i.e., w.r.t different sub-band)

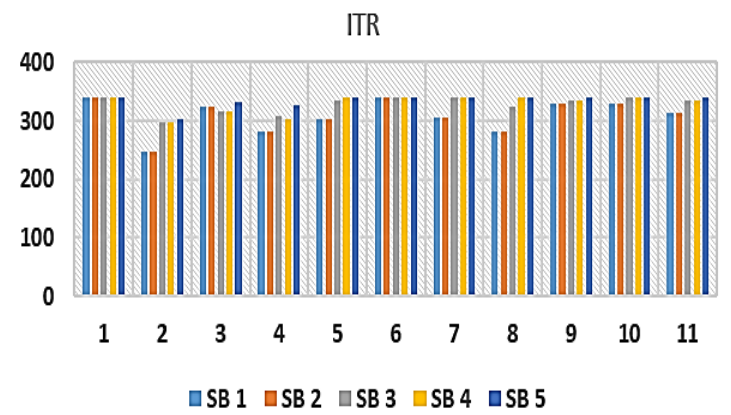

Figure 9. Simulated ITRs across subjects (i.e., w.r.t different sub-band)

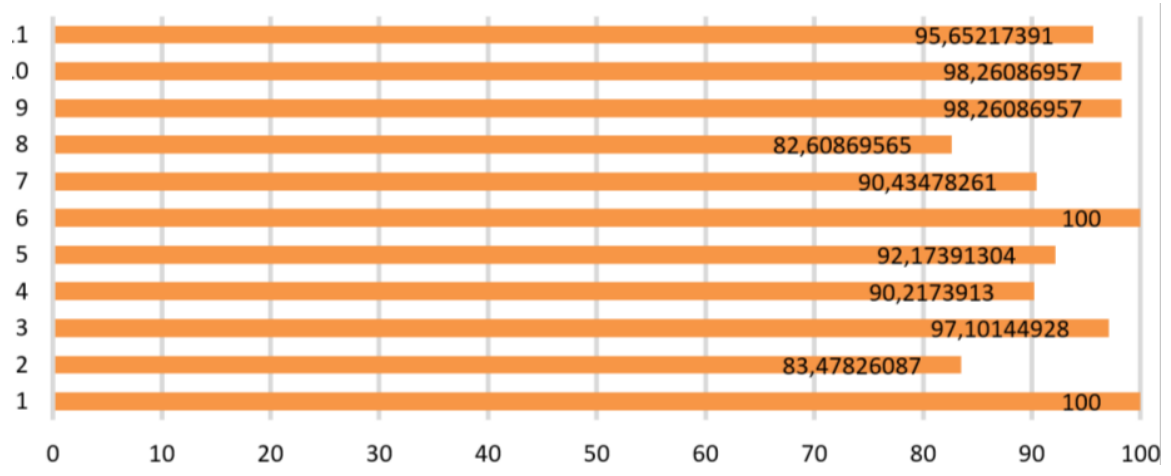

Figure 10. Averaged classification accuracy across subjects

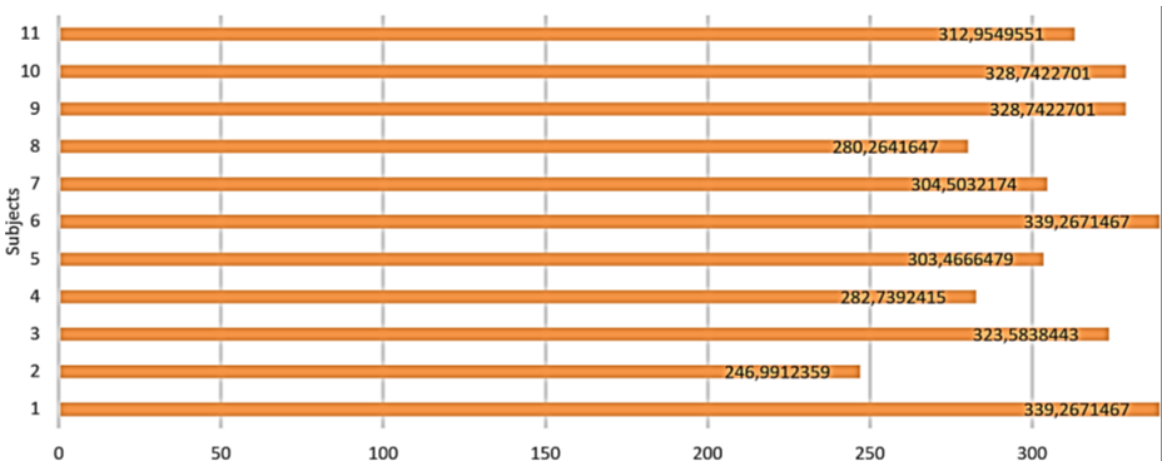

Figure 11. Simulated ITRs across subject 
Table 2. Results comparison with respect to state-of-art techniques

\begin{tabular}{cccccccc}
\hline \multirow{2}{*}{ Subject ID } & \multicolumn{3}{c}{ Accuracy } & \multicolumn{2}{c}{ ITR } \\
\cline { 2 - 7 } & Decision Trees & Ensemble Approach & LDA & OES & SVM & EDCA \\
\hline S-1 & 69.56 & 91.3 & 95.65 & 97.1014 & 98.55 & 100 & 339.2671 \\
S-2 & 65.21 & 82.6 & 73.04 & 99.1304 & 87.82 & 83.47826 & 246.9912 \\
S-3 & 28.98 & 34.78 & 27.53 & 52.1739 & 34.78 & 97.10145 & 323.5838 \\
S-4 & 50 & 71.73 & 64.13 & 78.2609 & 77.17 & 90.21739 & 282.7392 \\
S-5 & 24.34 & 25.21 & 19.13 & 27.8261 & 30.43 & 92.17391 & 303.4666 \\
S-6 & 50.43 & 82.6 & 79.13 & 97.3913 & 86.08 & 100 & 339.2671 \\
S-7 & 58.26 & 66.95 & 48.69 & 89.5652 & 60 & 90.43478 & 304.5032 \\
S-8 & 20.28 & 20.28 & 23.18 & 36.2319 & 31.88 & 82.6087 & 280.2642 \\
S-9 & 77.39 & 98.26 & 98.26 & 100 & 100 & 98.26087 & 328.7423 \\
S-10 & 52.17 & 86.95 & 78.26 & 97.3913 & 92.17 & 98.26087 & 328.7423 \\
S-11 & 63.47 & 82.6 & 98.26 & 99.1304 & 98.26 & 95.65217 & 312.955 \\
Mean & 50.91727 & 67.56909 & 64.11455 & 79.47298 & 72.46727 & 93.47167 & 308.2293 \\
\hline
\end{tabular}

Moreover, the classification accuracy and ITR results is also shown in the proposed EACA approach, where it is clearly seen that in most of subject better outcome has been achieved compared to other existing approaches. A more graphical intuitive result is represented in Figure 12 for classification accuracy comparison with respect to state-of-art techniques.

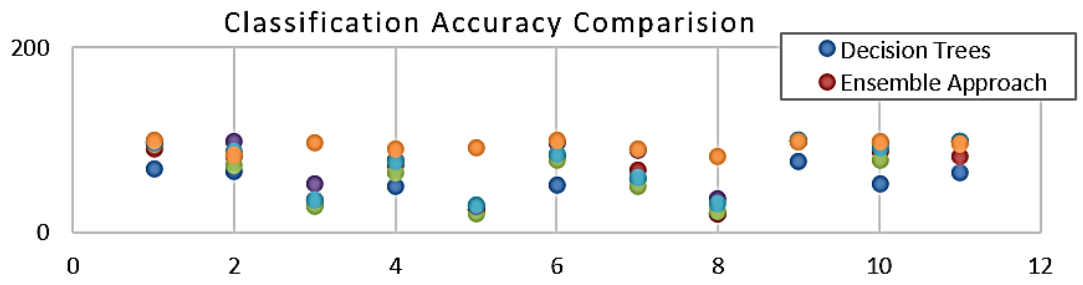

Figure 12. Classification accuracy comparison with respect to state-of-art techniques

\section{CONCLUSION}

The proposed EACA methods have shown great potential to optimize the classification accuracy of high-frequency-SSVEPs, also the EACA methods based spatial filtering is applied to decrease the noises of other EEG components. This plays significant role in the BCI, clinical applications, and cognitive neuroscience field. The publicly available dataset is considered to successfully conduct the experiment, where it shows result analysis in terms of different trails and subjects. In addition, the different existing methodologies has been considered in order to compare with proposed methodology. It is analyzed that EACA approach has got the $93.47 \%$ mean classification accuracy from 11-subjects that were considered, which is $14.97 \%$ and $22.47 \%$ more compared to OES and SVM approach, respectively. As per this analysis it can concluded that the proposed EACA based approach has performed well for classification result which is followed by this feature extraction process.

\section{REFERENCES}

[1] J. J. Shih, D. J. Krusienski, and J. R. Wolpaw, "Brain-computer interfaces in medicine," Mayo Clinic Proceedings, vol. 87, no. 3, 2012, pp. 268-279, 2012, doi: 10.1016/j.mayocp.2011.12.008.

[2] J. R. Wolpaw, N. Birbaumer, D. J. McFarland, G. Pfurtscheller, and T. M. Vaughan,., "Brain-computer interfaces for communication and control," Clinical Neurophysiology, vol. 113, no. 6, pp. 767-791, 2002, doi: $10.1016 /$ S1388-2457(02)00057-3.

[3] S. Gao, Y. Wang, X. Gao, and B. Hong, "Visual and auditory brain- computer interfaces," IEEE Engineering in Medicine and Biology Society, vol. 61, no. 5, pp. 1436-1447, 2014, doi: 10.1109/TBME.2014.2300164.

[4] J. F. Valenzuela-Valdes, M. A. Lopez, P. Padilla, J. L. Padilla, and J. Minguillon, "Human neuro-activity for securing body area networks: Application of brain-computer interfaces to people-centric Internet of Things," IEEE Communications Magazine, vol. 55, no. 2, pp. 62-67, 2017, doi: 10.1109/MCOM.2017.1600633CM.

[5] S. Movassaghi, M. Abolhasan, J. Lipman, D. Smith, and A. Jamalipour, "Wireless body area networks: A survey," IEEE Communications surveys and tutorials, vol. 16, no. 3, pp. 1658-1686, 2014, doi: 10.1109/SURV.2013.121313.00064.

[6] A. F. Demir et al., "In vivo communications: Steps toward next generation of implantable devices," IEEE Vehicular Technology Society, vol. 11, no. 2, pp. 32-42, 2016, doi: 10.1109/MVT.2016.2520492. 
[7] A. F. Demir et al., "Anatomical region-specific in vivo wireless communication channel characterization," IEEE journal of biomedical and health informatics, vol. 21, no. 5, pp. 1254-1262, 2017, doi: 10.1109/JBHI.2016.2618890.

[8] M. Wang, R. Li, R. Zhang, G. Li, and D. Zhang, "A wearable SSVEP-based BCI system for quadcopter control using head-mounted device," IEEE Access, vol. 6, pp. 26789-26798, 2018, doi: 10.1109/ACCESS.2018.2825378

[9] F. B. Vialatte, M. Maurice, J. Dauwels, and A. Cichocki, "Steady-state visually evoked potentials: Focus on essential paradigms and future perspectives," Prog. Neurobiol., vol. 90, no. 4, pp. 418-438, 2010, doi: 10.1016/j.pneurobio.2009.11.005

[10] C. S. Herrmann, "Human EEG responses to 1-100 Hz flicker: Resonance phenomena in visual cortex and their potential correlation to cognitive phenomena," Experimental Brain Research, vol. 137, no. 3-4, pp. 346-353, 2001, doi: $10.1007 / \mathrm{s} 002210100682$.

[11] Y. Wang, R. Wang, X. Gao, B. Hong, and S. Gao, "A practical VEP based brain-computer interface," IEEE Transactions on neural systems and rehabilitation engineering, vol. 14, no. 2, pp. 234-240, 2006, doi: 10.1109/TNSRE.2006.875576

[12] Z. Lin, C. Zhang, W. Wu, and X. Gao, "Frequency recognition based on canonical correlation analysis for SSVEPbased BCIS," IEEE transactions on biomedical engineering, vol. 53, no. 12, pp. 2610-2614, 2006, doi: 10.1109/TBME.2006.886577.

[13] H. Bakardjian, T. Tanaka, and A. Cichocki, "Optimization of SSVEP brain responses with application to eightcommand brain-computer interface," Neuroscience letters, vol. 469, no. 1, pp. 34-38, 2010, doi: 10.1016/j.neulet.2009.11.039.

[14] Y. Zhang, G. Zhou, J. Jin, M. Wang, X. Wang and A. Cichocki, "L1-regularized multiway canonical correlation analysis for SSVEP-based BCI," IEEE transactions on neural systems and rehabilitation engineering, vol. 21, no. 6, pp. 887-896, 2013, doi: 10.1109/TNSRE.2013.2279680.

[15] X. Chen, Y. Wang, S. Gao, T.-P. Jung, and X. Gao, "Filter bank canonical correlation analysis for implementing a high-speed SSVEP-based brain-computer interface," Journal of neural engineering, vol. 12, no. 4, Art. No. 046008, 2015.

[16] H. Wang et al., "Discriminative feature extraction via multivariate linear regression for SSVEP-based BCI," IEEE Transactions on Neural Systems and Rehabilitation Engineering, vol. 24, no. 5, pp. 532-541, 2016, doi: 10.1109/TNSRE.2016.2519350.

[17] M. Bittencourt-Villalpando and N. M. Maurits, "Stimuli and feature extraction algorithms for brain-computer interfaces: A systematic comparison," IEEE Transactions on Neural Systems and Rehabilitation Engineering, vol. 26, no. 9, pp. 1669-1679, 2018, doi: 10.1109/TNSRE.2018.2855801.

[18] K. Georgiadis et al., "Mamem EEG SSVEP dataset i (256 channels, 11 subjects, 5 frequencies)," 2018, doi: 10.6084/m9.figshare.2068677.v1.

[19] Y. J. Kim, N. S. Kwak and S. W. Lee, "Classification of motor imagery for Ear-EEG based brain-computer interface," in $6^{\text {th }}$ International Conference on Brain-Computer Interface (BCI), GangWon, Korea (South), 2018, pp. 1-2, doi: 10.1109/IWW-BCI.2018.8311517

[20] P. Prashant, A. Joshi, and V. Gandhi, "Brain computer interface: A review," IN 2015 5th Nirma University International Conference on Engineering (NUiCONE), 2015, pp. 1-6, doi: 10.1109/NUICONE.2015.7449615.

[21] M. N. I. Qureshi, D. Cho, and B. Lee, "EEG classification for motor imagery BCI using phase-only features extracted by independent component analysis," in 2017 39th Annual International Conference of the IEEE Engineering in Medicine and Biology Society (EMBC), Seogwipo, 2017, pp. 2097-2100, doi: 10.1109/EMBC.2017.8037267.

[22] M. Mohammadpour, S. M. R. Hashemi and N. Houshmand, "Classification of EEG-based emotion for BCI applications," in 2017 Artificial Intelligence and Robotics (IRANOPEN), 2017, pp. 127-131, doi: 10.1109/RIOS.2017.7956455.

[23] N. Yildirım and A. Varol, "A research on estimation of emotion using EEG signals and brain computer interfaces," In 2017 International Conference on Computer Science and Engineering (UBMK), Antalya, 2017, pp. 1132-1136, doi: 10.1109/UBMK.2017.8093523.

[24] C. Deng, J. Tong, X. Deng, Z. Zhang, and Y. Qin., "Emotion Recognition Positively Correlates with Steady-state Visual Evoked Potential Amplitude and Alpha Entrainment," Journal of Neuroscience, vol. 434, pp. 191-199, 2020, doi: 10.1016/j.neuroscience.2020.01.045.

[25] V. P. Oikonomou et al., "Comparative evaluation of state-of-the-art algorithms for SSVEP-based BCIs," arXiv preprint arXiv:1602.00904, 2016. 